\title{
Summary of the American College for Advancement in Medicine May 2005 Conference
}

\section{Menopause, Andropause: Power in Transition}

\section{Stephen A. Feig ${ }^{1}$, Eleanor Hynote ${ }^{2}$, Neal Speight ${ }^{3}$, Allan Magaziner ${ }^{4}$, Ralph A. Miranda ${ }^{5}$ and Michael B. Schachter ${ }^{6}$}

\author{
${ }^{1}$ Integrative Medical Consulting, 208 Vista Bella Drive, Santa Cruz, CA 95060, ${ }^{2}$ Phoenix Wellcare, 935 Trancas \\ Street Suite 1A, Napa, CA 94558, ${ }^{3}$ The Center For Wellness, 2317 Randolph Road, Charlotte, NC 28207, ${ }^{4}$ University \\ of Medicine and Dentistry of New Jersey, Robert Wood Johnson Medical School, 1907 Greentree Road, Cherry Hill, \\ NJ 08003, and ${ }^{5}$ Wholistic Health Center, Laser Surgery Associates, The ECP Center, RR \#12 Box 108, Greensburg, \\ PA 15601 and ${ }^{6}$ Schachter Center for Complementary Medicine, 2 Executive Boulevard, Suite 202, Suffern, \\ NY 10901, USA
}

The American College for Advancement in Medicine's (ACAM) 63rd International Educational Symposium on Complementary, Alternative and Integrative Medicine (CAIM) was held on May 18-22, 2005 at Disney's Coronado Springs Resort in Orlando, FL, USA. ACAM's scientific symposium brought together physicians and scientists from 13 countries for a comprehensive educational program on the evaluation and treatment of age-associated hormone deficiency with special attention to the use of bioidentical hormones in andropause and menopause.

ACAM's Spring 2005 convocation, entitled 'Menopause, Andropause: Power in Transition', addressed a multitude of topics relevant for physicians and scientists interested in improving their understanding of safe and effective hormone replacement therapy, nutritional intervention and the lifestyle modifications needed in order to ensure healthier and longer lives as our population transitions into middle age. The scientific presentations focused upon the use of bioidentical hormone supplementation for the treatment of aging and a wide variety of illnesses. Keynote presentations highlighted the adverse consequences of endocrine disrupting chemicals on human health along with recent advances in predictive genomics testing for evaluating both risks and benefits of hormone replacement therapy. Evidence-based protocols were presented for the use of bioidentical hormones in the treatment of

For reprints and all correspondence: Stephen A. Feig, D.O. c/o The American College for Advancement in Medicine, 23121 Verdugo Drive, Suite 204,

Laguna Hills, CA 92653, USA. Tel: +949-583-7666; Fax: +949-455-9679;

E-mail: info@acam.org disorders of the endocrine, reproductive and cardiovascular systems.

As a growing body of research has demonstrated a significant increase in disease risk in menopausal women taking conventional non-bioidentical hormone replacement therapy $(1,2)$, the conference served as a testament to the overwhelming professional interest in the use of bioidentical hormones and CAIM therapies for treating disorders of hormonal senescence. Over 350 physicians and other health professionals convened in Orlando at ACAM's 'Menopause, Andropause: Power in Transition' conference, in order to learn about the latest science behind the use of cutting edge CAIM protocols and bioidentical hormones in the practice of integrative medicine. ACAM's President, Dr Allan Magaziner commented, 'We had a record number of new attendees at this convention and their level of satisfaction and desire to attend future meetings was astounding. I encourage any forwardthinking physician or health care practitioner to attend the November 2005 ACAM meeting in Anaheim since it, too, promises to offer the highest level of practical applications and education in alternative, complementary and integrative medicine'.

A complete list of conference presentations can be found following this article.

In addition to the many exciting presentations at the scientific conference, ACAM offered in-depth 2 day preconference workshops on Mesotherapy and on Heavy Metal Toxicity along with a 1 day workshop on Scientific Writing.

Edwin Cooper, PhD, Distinguished Professor, Department of Neuroimmunology, at the David Geffen School of Medicine

(C) The Author (2005). Published by Oxford University Press. All rights reserved.

The online version of this article has been published under an open access model. Users are entitled to use, reproduce, disseminate, or display the open access version of this article for non-commercial purposes provided that: the original authorship is properly and fully attributed; the Journal and Oxford University Press are attributed as the original place of publication with the correct citation details given; if an article is subsequently reproduced or disseminated not in its entirety but only in part or as a derivative work this must be clearly indicated. For commercial re-use, please contact journals.permissions@oupjournals.org 
at University of California, Los Angeles, chaired ACAM's first Writer's Workshop held for those interested in developing their scientific writing skills. It was well attended and quite helpful in developing a foundational perspective on the art and science of writing for the peer reviewed scientific literature. Several participants brought their work for review and received constructive advice on optimizing their presentations for submission to medical journals. ACAM will be repeating this important workshop preceding the November 2005 meeting in Anaheim, CA, USA.

ACAM's president Dr Allan Magaziner chaired ACAM's popular workshop on 'Introducing Aesthetic Mesotherapy Into Your Practice'. Mesotherapy was originally developed in France by Dr Michel Pistor and is currently being administered by more that 15000 doctors in France. Mesotherapy involves the injection of vitamins, minerals, amino acids, homeopathics and other biologicals, and can help with body sculpting, weight loss, cellulite reduction, wrinkle reduction and facial rejuvenation.

Peter A. Salas, MD, a Board Certified Plastic Surgeon, presented data from his studies, which supported the use of mesotherapy as a valuable and effective treatment in aesthetic medicine. In research presented at meetings of the American Society for Aesthetic Plastic Surgery and the American Society of Plastic Surgeons (ASPS), Dr Salas demonstrated reduction in abdominal fat as measured by improvement in abdominal circumference and pinch test compared with placebo. There was a high degree of patient satisfaction. He also noted that a combination of therapeutic agents was superior to a single agent. Additionally, in patients who underwent treatment of lower eyelid fat pads, $90 \%$ of patients reported improvement. Dr Salas is conducting additional studies to be presented at a future ASPS meeting. ACAM is the first medical organization to teach a combination of classical mesotherapy, biomesotherapy and lipodissolve at one conference.

In the 'Heavy Metal Toxicity Workshop', groundbreaking progress was made in developing a consensus among leading physicians and scientific experts regarding provocative urine challenge testing protocols using multiple chelating agents for maximal diagnostic reliability. The Workshop Chairman, Ralph Miranda, MD, stated, 'We made a definite leap in our understanding of the breadth of toxic effects attributable to metals in the wrong tissue at the wrong levels at the wrong time, as well as the wide array of therapeutic options we can use to accelerate removal and mitigate toxic effects of metals'. The 'Heavy Metal Toxicity Workshop' educated participants about the emerging biochemical and pathologic 'smoking guns' causally linking Alzheimer's type dementia, autistic spectrum disorders, autoimmune disorders and other clinical challenges with the detrimental effects of toxic metals. The exchanges among participants and faculty were stimulating and resulted in great progress in the understanding of laboratory diagnostics and the treatment of metal toxicity.

On the final day of the conference, ACAM's Conference Co-Chair Eleanor Hynote, MD, presented Alexander Schauss,
$\mathrm{PhD}$, with the 2005 Linus Pauling Lecture Award in recognition of his outstanding contributions to the medical sciences. Dr Schauss is the Director of Natural and Medicinal Products Research for the American Institute for Biosocial and Medical Research. The award is in honor of Dr Linus Pauling, the only person in the world to have won two unshared Nobel Prizes, in 1954 for Chemistry and in 1962 for Peace. 'Linus Pauling inspired me to go into research on nutrients and nutraceutical ingredients back in the 1970s, and I greatly respect and admire his work', stated Schauss.

For 25 years, Schauss has been the lead scientist for the American Institute for Biosocial and Medical Research, Inc., in Tacoma, WA, USA. He is also the author or co-author of more than 100 scientific papers in a diverse range of journals, and recently completed his 12 th book, on the subject of intraabdominal obesity in males. A former professor of natural products research and associate professor of research at two academic institutions, he is currently adjunct research professor of botanical medicine at the National College of Naturopathic Medicine in Portland, Oregon. Dr Schauss has served on several National Institute of Health advisory councils, been a reviewer of botanical monographs for the US Pharmacopeia Convention and the International Bibliographic Information on Dietary Supplements database (maintained through an interagency partnership with the Food and Nutrition Information Center), National Agricultural Library, NIH Office of Dietary Supplements and the US Department of Agriculture.

Anyone who missed the opportunity to attend ACAM's scientific conference 'Menopause, Andropause: Power in Transition' or the preconference workshops on 'Scientific Writing, Introducing Aesthetic Mesotherapy Into Your Practice' and/or the 'Heavy Metal Toxicity Workshop' can order tapes/CD's from http://www.acam.org or through telephone (+1-800-532-3688 or 949-583-7666).

The American College for Advancement in Medicine (ACAM) is a not-for-profit professional medical organization for physicians interested in learning about evidence-based CAIM therapies. ACAM's goals are to improve skills, knowledge and diagnostic procedures as they relate to complementary and alternative medicine; to support research; and to develop awareness of alternative methods of medical treatment. ACAM is dedicated to educating physicians and other health care professionals on the latest findings and emerging procedures in CAIM, botanical medicine, environmental medicine and in the use of effective evidence-based nutritional therapeutics. As the voice of CAIM, ACAM strives to increase awareness and recognition of complementary, alternative and integrative medical therapies, and play an active role in shaping the future of the CAIM movement.

ACAM represents nearly 1000 physicians in 30 countries and is the largest and oldest organization of its kind in the world dedicated to providing its members with educational excellence in integrative medicine. ACAM's leading edge educational programming facilitates the incorporation of clinically relevant biomedical therapies by fast-tracking scientific advancements 
in nutritional medicine, nutraceuticals and ethnopharmacology into the practice of cutting edge CAIM medicine.

ACAM was the first major medical organization to educate its members about such now well-accepted concepts as insulin resistance/metabolic syndrome, the detrimental effects of homocysteine, the protective effects of antioxidants, the clinical uses of essential fatty acids, cancer chemoprotection therapies with diet and herbs, testing for cardiosensitive C-reactive protein, the benefits of natural thyroid replacement and nutritional medicine as applied to psychiatry.

ACAM's next scientific conference 'Scientific Integrative Medicine: Advancing Health Horizons' will be held at the Anaheim Hilton from November 9-13, 2005. Workshop topics include a 2-day 'Nutrition Review and Certification' course in collaboration with the American College of Nutrition (approved for 16 CMEs), 'Prolotherapy', a 2-day course in partnership with the University of Wisconsin, Medical School (approved for 15 CMEs), 'Managing Heavy Metal Toxicity and Mitigating Disease' an updated 2-day course for physician, nurses and technicians, 'Hyperbaric Oxygen Therapy', a 2-day course in collaboration with the International Hyperbaric Association (approved for 15 CMEs), and ACAM's Scientific Writing Workshop (approved for 7.5 CMEs). For further information, please call ACAM at +1-800-532-3688 (in the USA) or +1-949-583-7666 (outside the USA) or register online at http://www.acam.org.

\section{Summary of Presentations}

\section{Thierry Hertoghe, MD}

Dr Hertoghe is the President of the World Society of Anti-aging Medicine and the European Academy of Quality of Life and Longevity Medicine. As the Co-Founder of the International Hormone Society and a third generation endocrinologist, he lectures all over the world on effective endocrine management in the aging patient.

\section{Treatment of Partial Growth Hormone, Testosterone and Cortisol Deficiencies in Adults}

Dr Hertoghe's review focused heavily on the clinical correlations between growth hormone (GH), testosterone, and cortisol deficiencies. Moreover, it highlighted the changes in physical appearance before and after GH replacement therapy and categorized two clinical syndromes of GH deficiency, which include clear improvement in lean body mass and resolution of anxiety and depression after initiation of therapy. $\mathrm{He}$ detailed the following benefits of GH replacement therapy: (i) reduction in total serum cholesterol, low-density lipoprotein and the cholesterol/high-density lipoprotein ratio after treatment with GH; (ii) decreased incidence of Crohn's disease; (iii) improvement in bone mineral density after 1 year of replacement therapy; (iv) better sleep; and (v) reduction in overall mortality rates and Myocardial Infarctions (MI) in hypopituitary patients who received growth hormone replacement therapy (GHRT).

Dr Hertoghe reviewed androgen and age-related diseases, detailing the Male Climacteric Syndrome and the benefit of androgen replacement therapy in this setting. He reviewed older literature, which showed that administration of dihydrotestosterone gel actually induced a $15 \%$ reduction in the size of the prostate. He detailed the connection between elevated estrogen levels in men and increase in prostate stroma. Dr Hertoghe took a similar approach in describing the clinical effects of cortisol with age-related diseases. He closed his lecture by reviewing the laboratory evaluation of androgen and cortisol deficiency, and the hormonal intervention needed to correct the described abnormalities.

\section{Michael Galitzer, MD}

Dr Galitzer is a board certified emergency medicine physician who practices integrative medicine in California, USA.

\section{The Bioenergetic Evaluation and Treatment of Menopause}

Dr Galitzer reviewed the various facets of menopause, noting that for proper hormonal functioning one must view the endocrine system as an integrated whole. He maintained that menopause was not only an issue of estrogen and progesterone (P4) deficiency but also related to adrenal, thyroid, pancreatic hormones, as well as testosterone. Additionally, he reviewed the concepts of metabolic typing and liver detoxification and the role they play in menopause and the general management of the ill patient. Objective evaluation of the hormonal axis and autonomic nervous system is achieved through the use of heart rate variability, a good history that includes assessment of environmental and emotional stressors to the adrenal gland, and proper use of the laboratory, including salivary, urine or blood hormone levels and biological terrain assessment. Therapeutic interventions were reviewed, including exercise, supplements, relaxation techniques, acupuncture and homeopathic interventions. Dr Galitzer's presentation was an excellent overview of the integrative approach to menopause and the factors leading to a chronically stressed endocrine system.

\section{Allan Warshowsky, MD, FACOG}

Dr Warshowsky is a board certified OBGYN who serves as an attending staff physician at New York's Beth Israel Medical Center and is the author of 'Healing Fibroids: A Doctors Guide to a Natural Cure'.

\section{Holistic Healing of Fibroid Tumors of the Uterus}

Dr Warshowsky reviewed an integrative approach to the assessment and treatment of fibroid tumors. He presented data showing that patients who received an integrative approach had a mean decrease in fibroid tumor size of $0.8 \mathrm{~cm}$ 
while those in the control group had an increase in size of $1.9 \mathrm{~cm}$. Dr Warshowsky emphasized the importance of proper diet, hormone balancing, good gastrointestinal and liver support, and the use of P4 and melatonin. Additionally, he detailed the use of herbal and homeopathic interventions, including Scudders Alterative, Echinacea, Fraxinus Ceanothus, Aconite, Phytolacca, Gelsemium, Vitex (chasteberry), Aurum Muriaticaum and Hydrastinum Muriaticum in the management of patients with fibroid tumors.

\section{Jeanette Graf, MD, FAAD}

Dr Graf is a board certified dermatologist in private practice. She previously served as an NIH research fellow in the area of extracellular matrix components.

\section{The Care of Aging Skin-from Cosmeceutical to Cosmolecular}

Dr Graf's presentation reviewed the transition of skin care products from cosmeceuticals to cosmoleculars and she made the point that skin rejuvenation would ultimately be achieved at the molecular level via altering signal transduction. Dr Graf reviewed the factors involved in skin aging, which typically originate with oxidative stress related to cigarette smoke, genetics, other environmental stressors, UVA and UVB.

Potential targets for skin rejuvenation therapies included collagen, collagen-degrading enzymes known as matrix metalloproteinases (MMPs) and skin membrane lipids. Relevant nutrients, which were discussed as potential agents for slowing the aging process of skin include: (i) topical vitamin $\mathrm{A}$, which appears to regulate gene transcription and inhibit MMPs and has been documented to increase collagen bundles resulting in dermal thickening, improved skin tone and reduced signs of photoaging; (ii) tocopherols, which are fat-soluble and known to neutralize free radicals in the cell membrane; (iii) vitamin $\mathrm{C}$, which functions as a tyrosinase inhibitor, a cofactor in hydroxylation of collagen and an inhibitor of MMP-1; (iv) idebenone, an analog of CoQ10; (v) alpha and beta hydroxy acids, which are used as exfoliants; (vi) tissue respiratory factors from yeast that promote cellular respiration and cell renewal; (vii) beta glucans; (viii) zinc, which functions as a cofactor for the antioxidant enzyme superoxide dismutase; (ix) copper, which serves as a promoter of collagen formation; and (x) polyphenols, which include flavonoids from soy, green tea catechins, carotenoids and procyanidins. Dr Graf concluded her presentation by making the essential point that synergy among nutrients was paramount for longterm skin management.

\section{T.S. Wiley}

T.S. Wiley is an anthropologist and the author of 'Sex, Lies, and Menopause'. She is a member of the New York Academy of Sciences and has been a guest investigator at Sansum Medical Research Institute.

\section{MultiPhasic, Cyclical Hormone Therapy with Bioidentical E2/P4}

Postulating that the cyclic hormone pattern and serum levels of perimenopausal and post-menopausal women should mimic that of women in their 20s, T.S. Wiley has generated a protocol with the use of transdermal bioidentical estradiol (E2) and P4 that is multiphasic and cyclic. Ms Wiley's presentation described the rational and early clinical experiences in using high-dose bioidentical hormone replacement therapy. The initial results of this therapy protocol have been positive and plans are underway to expand her clinical trial for statistical validation.

During the question and answer period, some concerns were raised about the protocol. These includes (i) whether or not serum levels were an acceptable marker for hormones that were administered transdermally; (ii) that some women had experienced significant side effects while following the protocol and had stopped using it; and (iii) the dosage of hormones used was too high and not physiologic.

\section{Lindsey Berkson, MA, DC}

Dr Berkson is presently a consulting scholar at the Center for Bioenvironmental Research at Tulane and Xavier Universities as well as the author of seven books on health and the environment.

\section{Endocrine Disruption Update and Relevance in Clinical Practice}

Dr Berkson reviewed the clinical relevance of endocrine disrupting chemicals. She discussed signal transduction and the impact of hormones and hormone mimics on gene transcription and suppression; specifically citing the similarities of di-ethyl-stilboestrol (DES), Dichlorodiphenyltrichloroethane (DDT) and bisphenol-A to E2. Dr Berkson's presentation reviewed the interplay, interdependence and downstream influence of various hormones upon one another and elegantly classified compounds known to be endocrine disruptors, which include: Polychlorinated biphenyls (PCBs), DDT, dioxins, pyrethroids, cadmium, lead, methoxychlor, etc.

The presentation briefly explained the mechanisms of action of many endocrine disrupters, which include competitive binding to the hormone binding globulins, interference with metabolic pathways of endogenous hormones, competitive binding to nuclear receptors, inhibition of the dimerization of the ligand-nuclear receptor complex, interference with the coupling between the dimer complex and the DNA response elements and the down-regulation of receptors.

\section{Eugene Shippen, MD}

Dr Shippen is the author of the best selling book, 'The Testosterone Syndrome'. 


\section{The Role of Testosterone in Disease and Aging}

Dr Shippen's lecture focused on the important role of testosterone in the aging process. He highlighted the many changes that occur with aging including the shift from $\mathrm{Th} 2$ to Th1 response and the resulting movement toward a more proinflammatory state. He emphasized that treatments with androgens, $\mathrm{P} 4$ and vitamin $\mathrm{D}$ tend to shift $\mathrm{T}$ lymphocytes from Th1 dominance back toward Th2 dominance thereby implying less inflammation. However, E2 results in excess shifts toward a more proinflammatory state. Additionally, he covered the potential role of MMP activity in the degradation and rupture of the fibrous cap of many vascular plaques. Interestingly, it appears that the activity of MMP is down-regulated in the presence of normal levels of testosterone and up-regulated in the presence of E2.

Dr Shippen made the point that many chronic diseases are associated with low testosterone and that treating these illnesses with androgen replacement therapy may offer relief. $\mathrm{He}$ also reviewed many classes of drugs that may lower testosterone, including antifungals, anticonvulsants, glucocorticoids, phenothiazines, calcium channel blockers, diuretics, statins and others.

\section{Joel Evans, MD}

Dr Evans is an assistant professor of both the Albert Einstein College of Medicine and the College of Physicians and Surgeons at Columbia University. He is also the Founder and Director of the Center for Women's Health in Darien, CT, USA.

\section{The Use of Genomic Testing to Assess Risks and Benefits of Hormone Replacement Therapy}

Many physicians are facing difficult decisions regarding initiating HRT. An individualized approach can include the use of predictive genomic testing. A considerable amount of research has been done to assess the effect of HRT on those who carry specific single nucleotide polymorphisms (SNPs). In many cases, the presence of a particular SNP can significantly increase or decrease a women's risk of complications from HRT. Certain SNPs can affect coagulation, such that women who are on HRT with the $\mathrm{G} \rightarrow \mathrm{A}$ polymorphism at position 20210A on chromosome 11 will have a higher risk for thromboembolic events. Genomic testing can also help to assess breast cancer risk in patients treated with HRT. CYP1A1 produces $2-\mathrm{OH}$ estrogens and decreased activity of this cytochrome leads to increased breast cancer risk. Similarly, CYP1B1 produces $4-\mathrm{OH}$ estrogens so that increased activity leads to increased ovarian and breast cancer risk.

\section{Eric Serrano, MD}

Dr Serrano is a Professor at Ohio State University.

\section{Hormones: What are they and What They Do}

Dr Serrano reviewed the general effects on hormone metabolism in relationship to obesity and explained how hormones, produced by the hypothalamus and the pituitary gland, are secreted in a burst-like fashion. Additionally, he reviewed the circadian rhythm of hormones such as GH and adrenocorticotrophin hormone (ACTH) and noted that these hormones have increased secretion during specific hours of the day. Circadian rhythms of hormone secretion have a direct influence on metabolism such that the first meal of the day has the ability to alter ones metabolic rate and ones ability to burn calories efficiently for the remainder of the day. Also, sex hormones influence metabolism such that the optimal time to exercise and burn the most calories is during the follicular phase of the menstrual cycle. Dr Serrano also noted that many obese patients have a higher accumulation of biotoxins and subsequently a 30\% lower ability to burn calories efficiently.

\section{Debu Tripathy, MD}

Dr Tripathy is Professor of Internal Medicine and Director of Komen, University of Texas Southwestern Breast Cancer Research Program at the University of Southwestern Medical Center in Dallas, TX, USA.

\section{Models of Research for Complementary Medicine in Breast Cancer}

Models of Integrative Cancer Research have included surveys (population-based, center-based and recruited), observational cohort studies and case-control studies. There are far fewer prospective and placebo-controlled trials. Dr Tripathy discussed his work in designing a 'Pilot Phase I Study of Tibetan Herbal Therapy for Advanced Breast Cancer'. Individuation rather than single agent therapy is necessary and the basis of choosing herbal formulae is not defined by standard clinical or scientific criteria. Dr Tripathy's study was designed to preserve the individualization of care by using the expertise of a Tibetan physician who determined treatment formulae based on history and physical examination. Eleven patients with minimally symptomatic measurable advanced breast cancer were treated with a Tibetan herbal formula as their only treatment. Assessments of tumor progression were performed and patients were treated until tumor progressed. The results revealed that none of the patients had significant toxicities. One of the nine patients had a significant partial response after 4 months. One patient had stable disease for greater than 1 year.

\section{Mark Blumenthal}

Mark Blumenthal is the Founder and Executive Director of the American Botanical Council; an independent, non-profit organization dedicated to disseminating accurate, reliable and responsible information on herbs and medicinal plants. He is the Editor/Publisher of HerbalGram, an international, 
peer-reviewed quarterly journal. For 6 years he served as an Adjunct Associate Professor of Medicinal Chemistry at the College of Pharmacy, University of Texas at Austin. Mark has served as Co-Founder and former Vice President of the Herb Research Foundation and President of the Herb Trade Association. He was also a founding board member of the American Herbal Products Association. He is the senior editor of the English translation of The Complete German Commission E Monographs-Therapeutic Guide to Herbal Medicines, a rational system for evaluating the safety and efficacy of herbal medicines. Mark is the senior editor of The American Botanical Council's Clinical Guide to Herbs, a new reference work and continuing education module.

\section{Review of the Research on Botanical Medicines for Treating the Symptoms of Menopause and 'Manopause'}

Mr Blumenthal reviewed the clinical evidence and the safety data for the use of black cohosh, red clover extract and soy isoflavones in the treatment of menopausal symptoms. Studies documenting the safety and efficacy of saw palmetto, pygeum bark and nettle root were discussed in relationship to assisting men with the symptoms of andropause.

\section{Dennis Villareal, MD}

Dr Villareal is Assistant Professor of Medicine in Geriatrics and Nutritional Science and Director of Geriatric Consultation Service at the Barnes-Jewish Hospital at Washington University.

\section{DHEA and the Metabolic Syndrome}

Dr Villareal made the following points in regards to his original research on Dehydroepiandrosterone (DHEA): (i) the adrenal production of DHEA declines dramatically with aging in humans. Low DHEA levels have been correlated with abdominal obesity, glucose intolerance, insulin resistance and atherosclerosis; (ii) higher DHEA levels have been correlated with longer survival; and (iii) DHEA is the most abundant hormone in the human body.

His research addressed the question of whether DHEA replacement is beneficial. Dr Villareal presented his data showing that there is a measurable decrease in visceral fat with DHEA replacement in elderly women and men. This decrease in adipose tissue will result improved insulin resistance. Although there was no impact of DHEA supplementation on testosterone levels in men, women who were supplemented with DHEA showed an increase of testosterone, which mimicked levels that are more youthful. Dr Villareal's research also revealed a slight increase in insulin-like growth factor-1 (IGF-1) in both women and men supplemented with DHEA. There were no serious adverse effects related to DHEA replacement. No changes in liver and renal chemistries were observed and, in fact, there was a slight downward trend in PSA levels in men.

\section{Alexander Schauss, PhD}

Dr Schauss is the author of 'Minerals, Trace Elements and Human Health' and serves as the Director of Natural and Medicinal Products Research at AIBMR Life Sciences, Inc.

\section{Minerals: Achieving the Proper Balance}

Recent research indicates that supplemental minerals and trace elements may play a significant role in maintaining health and preventing disease. Nevertheless, they must exist in balance and ingestion of these nutrients does not come without risk in some cases when there is a lack of safety data and toxicology. Dr Schauss discussed the potential harmful effect of chronic germanium ingestion and potential toxicity of vanadium supplementation. Of particular concern is the accumulation of vanadium into the kidneys and other tissues. Dr Schauss pointed out that safer forms of vanadium supplements, such as vanadyl-methylpicolinate complex, are now available. He also discussed the importance of selenium as a trace mineral and indicated that depletion of selenium is known to cause disease, especially with impairment of the immune system. Selenomethionine was discussed as the best source for human ingestion.

\section{Cynthia Browne, MD, PhD}

Dr Brown is Director of Complementary Cancer Care at the Van Elslander Cancer Center located in Gross Point Woods, MI, USA.

\section{Estrogen Metabolic Pathways: the Road to Health or Disease}

Dr Brown's presentation examined how various toxins, exotoxins, hormones and drugs are processed by the liver in a two-step process of Phase I and Phase II detoxification. She described the various forms of estrogen (E1, E2 and E3) and the complex interaction with other hormones on their path to metabolism and excretion. Variations in p450 enzymes in Phase I detoxification can affect the ratio of E2 and estrone 2-OH, 16 alpha-hydroxy and 4-hydroxy metabolites. These have implications for health and disease as further metabolites through Phase II conjugation have varying properties of biochemical significance. Dr Brown explained that the ultimate effect of estrogen depends on how it is metabolized, and that genetics and nutrients dictate estrogen metabolism through methylation, glucuronidation and sulfation. The contribution of cancer risk is from total estrogen load as well as types of metabolites. Estrogen metabolism is also influenced by aromatase activity. Higher aromatase levels increase estrogen load and some of the factors that increase aromatase include obesity and inflammation. Dr Brown explained how nutritional interventions influence estrogen metabolism and physiology. Although the efficacy of indole-3 carbinol depends on stomach pH to convert to Diindolemethane (DIM), both nutrients have similar beneficial effects of improving 2-OH estrone to 16 alpha-OH estrone ratios. It appears that not all beneficial 
effects are from DIM as other metabolites from 13C, such as indolylcarbazole, can induce CYP1A1 and CYP13C but have a greater effect on CYP3A4 than DIM in vivo.

\section{John Kells, MD}

Dr Kells is CEO of Aeron Labs.

Salivary Bioavailable Testosterone (SBA-T) As

Compared To Serum Bioavailable (BT), Total

Testosterone (TT) and Calculated Free Testosterone Index (cFT)

Dr Kells performed a study at St Louis University, which compared SBA-T with BT and TT levels in 127 men aged 23-80 years. He found that SBA-T was highly significantly correlated with BT $(P<0.001)$. Dr Kells noted that testosterone appeared to be a biomarker for longevity in women but its significance as a predictor of longevity in men was uncertain. The conclusion of Dr Kells study was that his data support the legitimacy of using SBA-T to measure bioeffective, tissue available testosterone and that SBA-T was clinically useful in the diagnosis of hypogonadism.

\section{References}

1. Cauley JA, Robbins J, Chen Z, et al. Effects of estrogen plus progestin on risk of fracture and bone mineral density: the Women's Health Initiative randomized trial. J Am Med Assoc 2003;290:1729-38.

2. Rossouw JE, Anderson GL, Prentice RL, et al. Risks and benefits of estrogen plus progestin in healthy postmenopausal women: Principal results from the Women's Health Initiative randomized controlled trial. J Am Med Assoc 2000;288:321-33. 


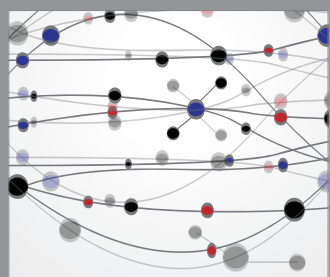

The Scientific World Journal
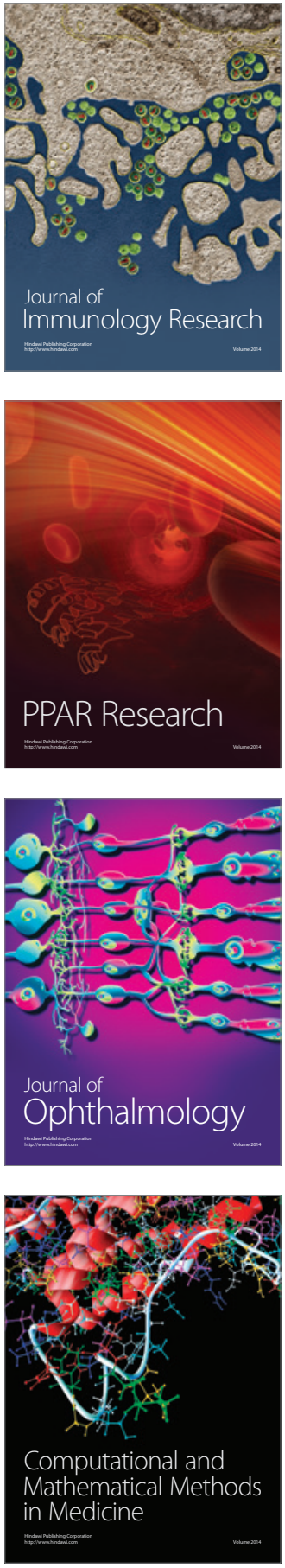

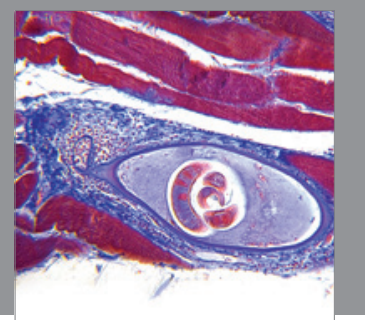

Gastroenterology

Research and Practice
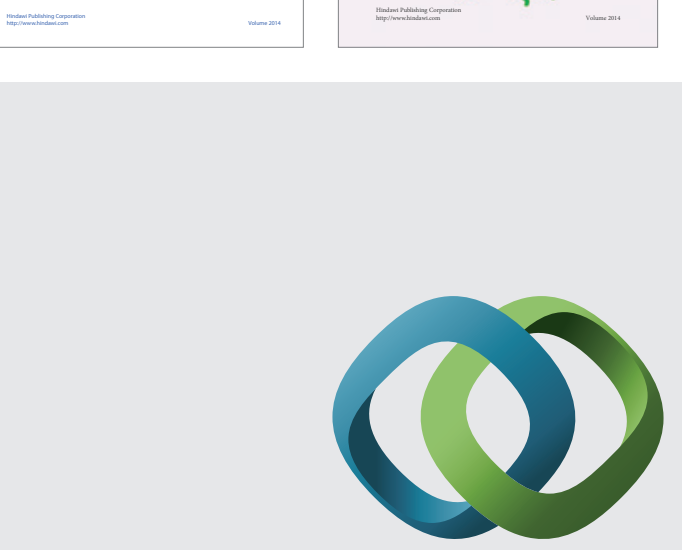

\section{Hindawi}

Submit your manuscripts at

http://www.hindawi.com
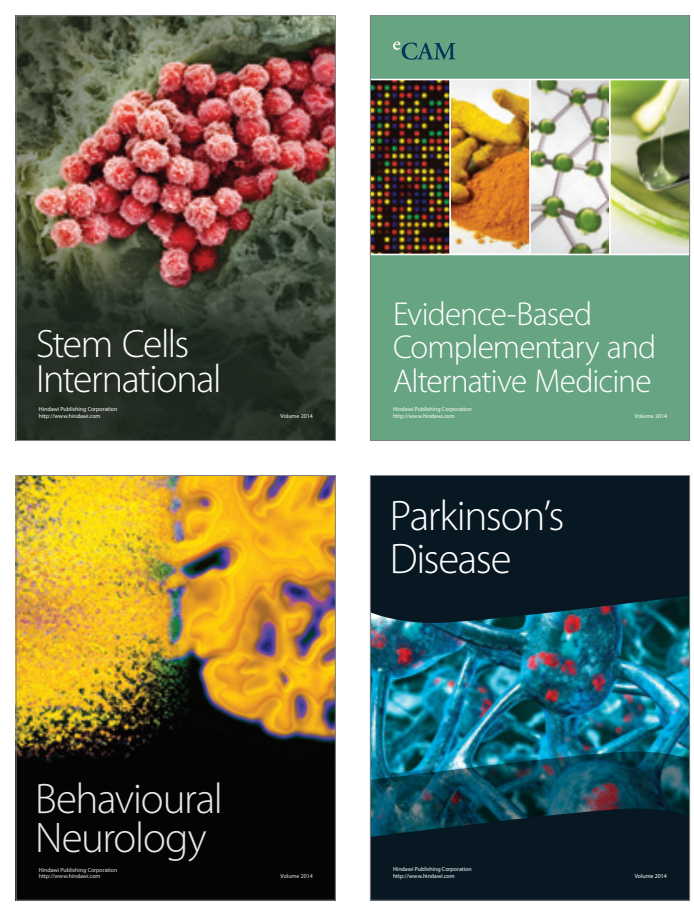

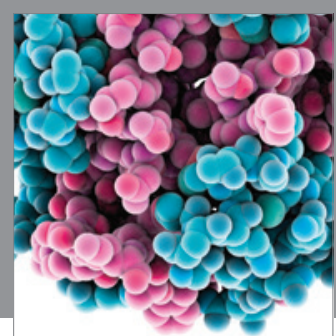

Journal of
Diabetes Research

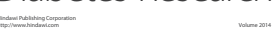

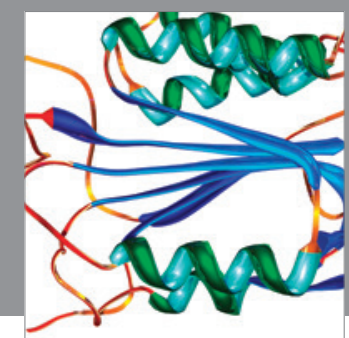

Disease Markers
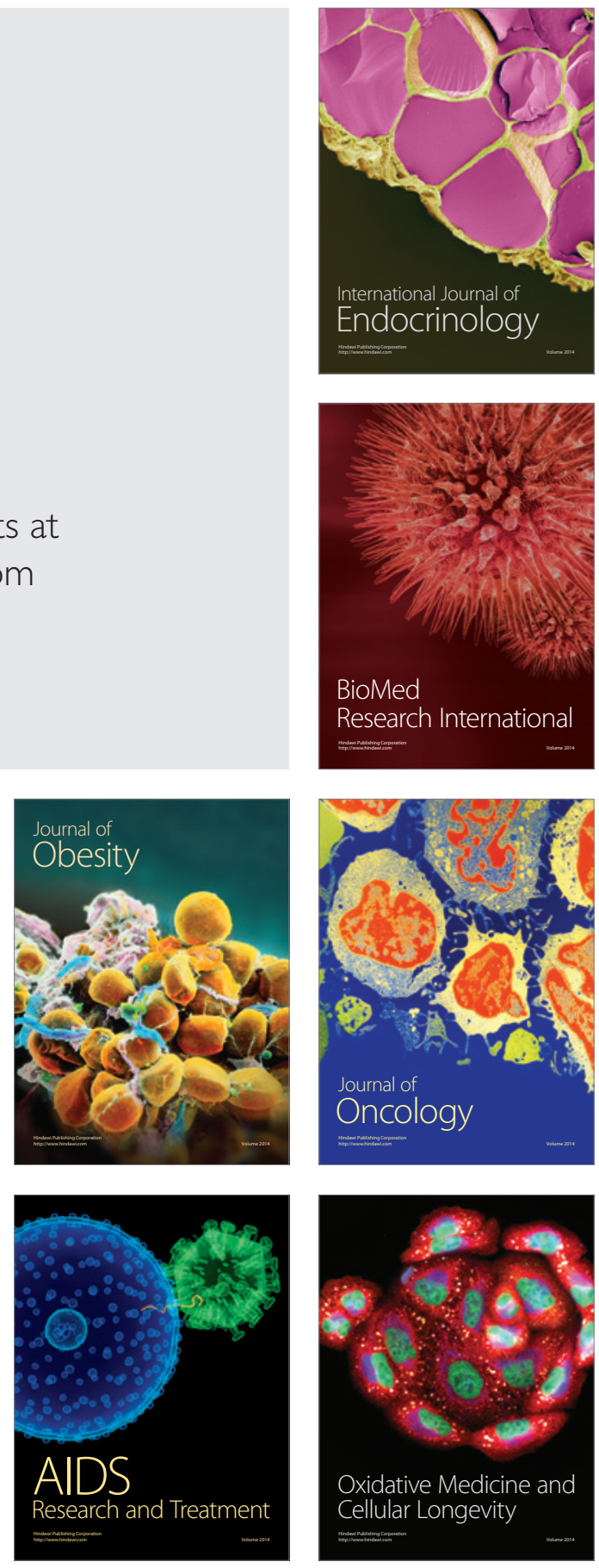